\title{
Randomized Embolization Trial for NeuroEndocrine Tumor Metastases to the Liver (RETNET): study protocol for a randomized controlled trial
}

\author{
James X. Chen ${ }^{1}$, E. Paul Wileyto ${ }^{2,3}$ and Michael C. Soulen ${ }^{1,3,4^{*}}$ (D)
}

\begin{abstract}
Background: Neuroendocrine tumors (NETs) are the second most common gastrointestinal malignancy after colon cancer. Up to $90 \%$ of patients with NETs develop liver metastases, which are a major determinant of symptoms and survival. Current guidelines recommend embolotherapy for progressive or symptomatic NET liver metastases, but the optimal technique among bland embolization, lipiodol chemoembolization, and drug-eluting bead chemoembolization remains unknown and controversial.
\end{abstract}

Methods/design: A prospective, open-label, multicenter randomized controlled trial will be conducted in patients with progressive or symptomatic unresectable NET liver metastases. Patients will be randomized to treatment with bland embolization, lipiodol chemoembolization, or drug-eluting microsphere chemoembolization, with 60 enrollees per arm. The primary endpoint will be hepatic progression-free survival (HPFS) following initial embolotherapy by RECIST criteria. The sample size is powered to detect an HR of 1.78 for HPFS following chemoembolization compared with bland embolization, which was estimated on the basis of existing retrospective studies. Secondary endpoints include overall progression-free survival, duration of symptom control, quality of life, rate of adverse events, and interval between embolotherapy cycles. Interim safety analyses will be performed at 10 and 30 patients per arm.

Discussion: The RETNET trial is a prospective, multicenter randomized controlled trial designed to determine the optimal embolotherapy technique for NET liver metastases.

Trial registration: ClinicalTrials.gov, NCT02724540. Registered on March 31, 2016.

Keywords: Neuroendocrine tumor, Embolization, Chemoembolization, Liver metastases

\section{Background}

Neuroendocrine tumors (NETs) are a heterogeneous family of neoplasms that arise from cells in the gastrointestinal (GI) tract, pancreas, and lung. With an estimated prevalence of $>100,000$ in the United States and a steadily increasing incidence over the past quarter century [1], NETs have become the second most common GI malignancy after colon cancer. NET cancer biology has a propensity for the development of liver metastases, which

\footnotetext{
* Correspondence: michael.soulen@uphs.upenn.edu

${ }^{1}$ Division of Interventional Radiology, University of Pennsylvania, Philadelphia, PA, USA

${ }^{3}$ Abramson Cancer Center, University of Pennsylvania, Philadelphia, PA, USA Full list of author information is available at the end of the article
}

occur in $40-90 \%$ of patients [2] and are a major determinant of symptoms and survival. Analysis of National Cancer Institute Surveillance, Epidemiology, and End Results registry data from 1973 to 2004 demonstrated that patients with localized, regional, or distant metastatic disease had median survivals of 223, 111, and 33 months, respectively [1]. With supportive care only, patients with unresectable liver metastases have a 5-year survival of 0 $22 \%$. Symptom control and survival outcomes improved in the late 1980s with the introduction of octreotide, a somatostatin analogue used to control hormone secretion-related symptoms. During the past three decades, there has also been widespread refinement and adoption of locoregional transarterial embolotherapy for

(c) The Author(s). 2018 Open Access This article is distributed under the terms of the Creative Commons Attribution 4.0 International License (http://creativecommons.org/licenses/by/4.0/), which permits unrestricted use, distribution, and 
treatment of NET liver metastases. By selectively embolizing hepatic arterial tumor feeders, metastases are cut off from their vascular supply, whereas the normal liver parenchyma retains viability via perfusion from the portal veins. Studies have demonstrated efficacy of these liver-directed embolotherapies for tumor growth reduction and NET symptom relief, with current 5-year survival rates of over $60 \%$ and response rates between $70 \%$ and $90 \%$ [3-13].

Liver-directed embolotherapy encompasses distinct techniques, including "bland" embolization (BE) using embolic particles instilled via the hepatic artery to cut off tumor blood supply and transarterial chemoembolization (TACE) using either an ethiodized oil emulsion of chemotherapeutic drugs (conventional lipiodol TACE, classical transarterial chemoembolization [CTACE]) or drugs loaded onto embolic microspheres (drug-eluting bead transarterial chemoembolization [DEB-TACE]). Current National Comprehensive Cancer Network, North American Neuroendocrine Tumor Society, and European Neuroendocrine Tumor Society guidelines support embolotherapy for symptomatic or progressive NET liver metastases on the basis of level IIB-3 evidence [14-18], but they offer no recommendation regarding available techniques.

Published studies comparing TACE with bland embolization (BE) have demonstrated heterogeneous and contradictory results, with some studies showing superior survival and/or safety outcomes for one technique $[5,6,9]$ and others demonstrating equivalent outcomes $[8,19,20]$. One of the potential contributors to these varying results was ambiguity in the World Health Organization (WHO) 2000/2004 NET pathologic grading systems, which were used in many older studies $[4,6,7,9,12,21]$ and have since been replaced by the more objective, reproducible WHO 2010 system. In addition to uncertainty about survival outcomes among embolotherapy techniques, the comparative safety profiles and treatment toxicities also remain controversial. Two retrospective series have suggested higher risks for biliary complications after DEB-TACE [13, 22], but there remains no definitive comparison with lipiodol TACE or BE. The combination of an indeterminate evidence base and lack of specific guideline recommendations have contributed to wide variations in clinical practice, manifesting in equivalent use of available embolotherapy techniques for NET liver metastases in a survey of U.S. interventional radiologists [23].

Given the increasing incidence of NET and ongoing questions regarding an optimal treatment algorithm for NET liver metastases, there is a need for a prospective randomized controlled trial (RCT) to elucidate the optimal embolotherapy technique between transarterial $\mathrm{BE}$ and chemoembolization. This article presents the study protocol of a prospective, open-label, multicenter RCT using a parallel group assignment and noninferiority design. The trial aims to determine the optimal embolotherapy modality for NET liver metastases by comparing hepatic progression-free survival (HPFS), treatment toxicities, symptom control, and quality of life.

\section{Methods/design Hypothesis}

The primary hypothesis of this study is that HPFS following TACE (lipiodol or DEB-TACE) will be nearly twice as durable as BE (HR, 1.78) for control of NET liver metastases. This will be tested against a null hypothesis of equality among embolization methods, without preconception of ranking.

\section{Study design}

This is an open-label, multicenter, randomized trial comparing three embolotherapy techniques for NET liver metastases: (1) BE, (2) cTACE, and (3) DEB-TACE. The study flowchart is shown in Fig. 1. Anticipated study sites include academic hospitals in the United States, Australia, Canada, France, and Argentina.

This trial will be conducted in accordance with the principles outlined in the Declaration of Helsinki and will follow the Consolidated Standards of Reporting Trials (CONSORT) statement. A Standard Protocol Items: Recommendations for Interventional Trials (SPIRIT) checklist is provided in Additional file 1.

\section{Study population}

Eligible participants will have liver-dominant NET that is symptomatic, progressive, or with a liver tumor burden $>25 \%$ of the liver volume without need for documented progression. Patients with extrahepatic disease progression can be enrolled in the study if they have liver-dominant disease based on the assessment of a multidisciplinary NET care team. Concurrent treatment with somatostatin analogues is allowed. No concomitant radiation therapy, chemotherapy (e.g., capecitabine/temozolomide), biologic therapy (e.g., everolimus, sunitinib), or ablation is allowed. Patients also cannot receive these treatments until after the primary endpoint of hepatic progression is reached. All participants will be consented with an institutional review board (IRB)-approved, site-specific informed consent form prior to undergoing any study-related procedures. Detailed inclusion and exclusion criteria are shown in Table 1. A SPIRIT figure is shown in Fig. 2, and a SPIRIT checklist is available in Additional file 1. Patients may be withdrawn from the study for failure to adhere to protocol requirements or withdrawal of consent. 
Patient screening and enrollment:

Anticipated accrual period of 24 months

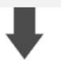

Randomization

$(1: 1: 1)$

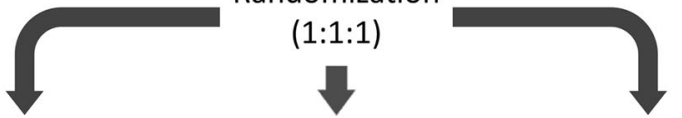

Arm 1

Arm 2

Arm 3

\begin{tabular}{c|c|c} 
Bland embolization & Lipiodol TACE & DEB TACE \\
$\mathrm{N}=60$ & $\mathrm{~N}=60$ & $\mathrm{~N}=60$
\end{tabular}

Embolotherapy (1 ${ }^{\text {st }}$ cycle)

Includes initial treatment and 94 week treatments to additional vascular territories until the entire tumor burden is addressed

Initial follow-up: 4-6 weeks following embolotherapy

Subsequent follow-up: Every 3 months, up to 24 months

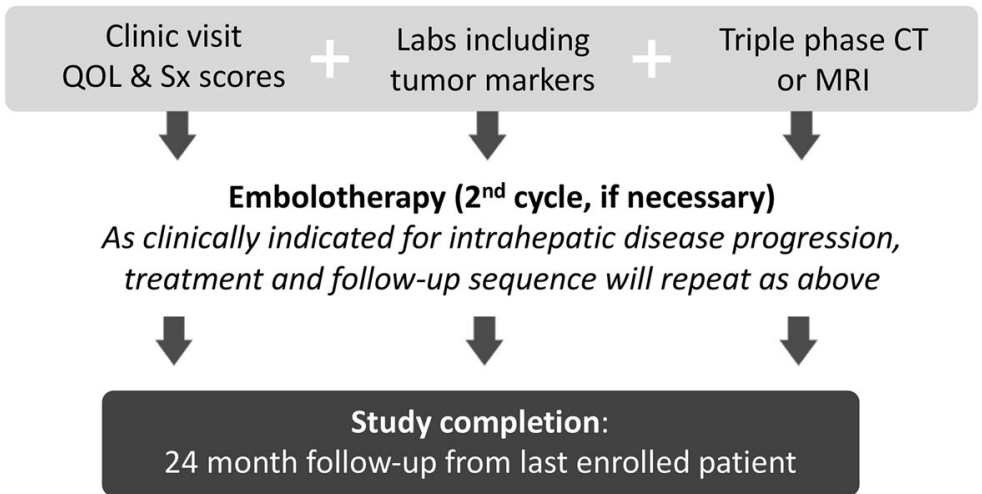

Fig. 1 Study flowchart. CT Computed tomography, MRI Magnetic resonance imaging, QOL Quality of life, Sx symptom

\section{Investigations and interventions \\ Baseline evaluation}

Clinical assessment, patient-reported NET quality of life (European Organization for Research and Treatment of Cancer [EORTC] QLQ-C30 and G.I.NET21 questionnaires) and Carcinoid Symptom Severity Score [24], screening laboratory studies (complete blood count, partial thromboplastin time, internal normalized ratio, comprehensive metabolic profile, tumor markers including chromogranin A), and imaging (abdominal computed tomography [CT] or magnetic resonance imaging [MRI], chest CT) will be performed within 30 days of initial embolotherapy. Patients with tumors without available histologic grading (WHO 2010) must undergo percutaneous biopsy to determine tumor grade. Once the eligibility data forms are completed in the ORACLE database (Oracle, Santa Clara, CA, USA) and baseline images are uploaded to the Imaging Core and undergo a quality control check, the treatment assignment is automatically generated via a built in block randomization program (see "Randomization" section below).

\section{Embolotherapy technique}

$\mathrm{BE}$ will be performed with lobar or segmental infusion of microspheres $(100-500 \mu \mathrm{m})$ to an embolization endpoint of two- to five-heartbeat stasis. Conventional lipiodol TACE will be performed with lobar or segmental infusion of doxorubicin $50 \mathrm{mg}$, dissolved in $10 \mathrm{ml}$ of diluted iodinated contrast agent, and emulsified with ethiodized oil, followed by embolization with 40$500-\mu \mathrm{m}$ microspheres. DEB-TACE will be performed with lobar or segmental infusion of drug-eluting microspheres $(100-300-\mu \mathrm{m}$ or $300-500-\mu \mathrm{m}$ microspheres loaded with doxorubicin per manufacturer's instructions for use). For all treatment arms, embolotherapy to additional vascular territories will be performed every 4 weeks until the entire tumor burden is treated. The decision for lobar or segmental embolization and 
Table 1 RETNET trial inclusion and exclusion criteria

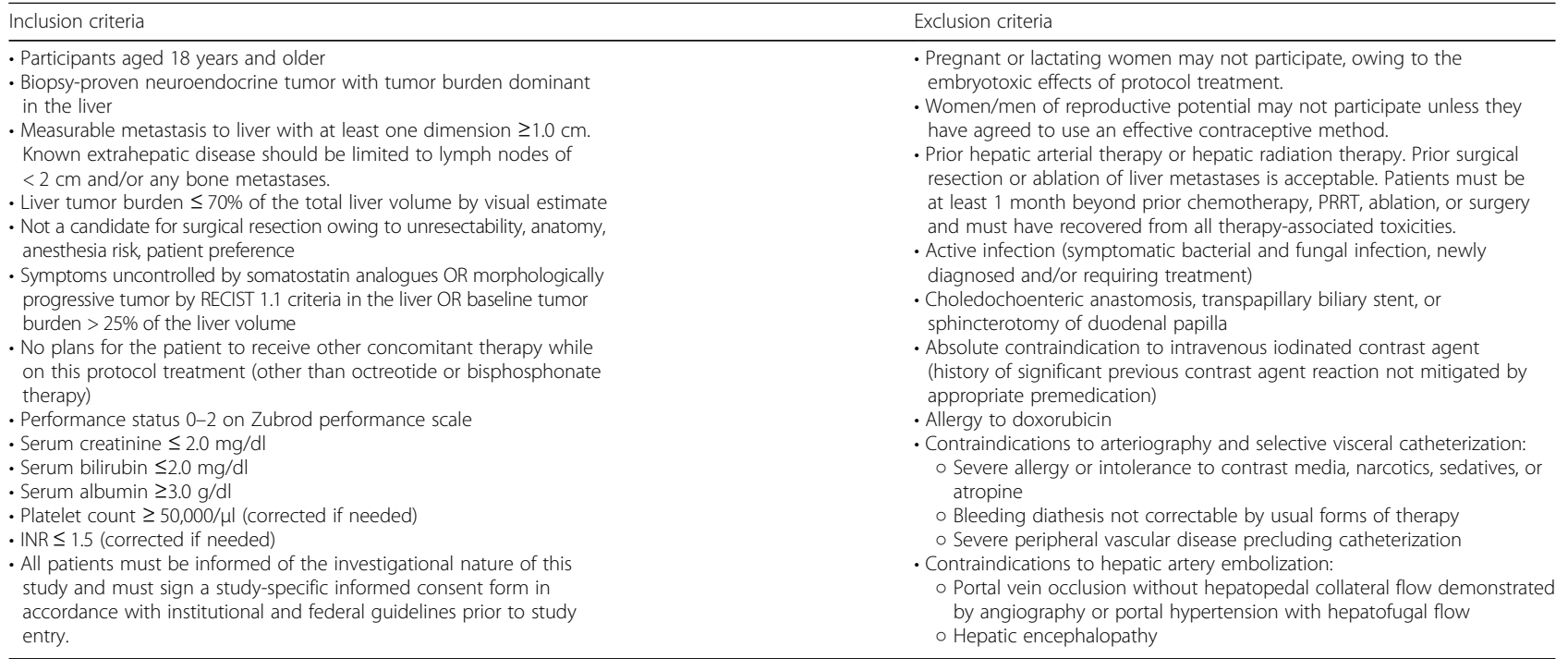

Abbreviations: INR International normalized ratio, PRRT Peptide receptor radionuclide therapy, RECIST Response Evaluation Criteria in Solid Tumors, RETNET Randomized Embolization Trial for NeuroEndocrine Tumor Metastases to the Liver

nuances of embolotherapy technique will be determined by operator preference.

\section{Follow-up evaluation}

Follow-up laboratory assays will be performed 33.5 weeks after the first embolotherapy session. If the patient remains eligible for further therapy, additional segmental or lobar embolization will be administered every 4 weeks until the entire disease area is adequately addressed. A maximum of four treatments can be performed per treatment cycle.

After the final embolotherapy session of the treatment cycle, serial clinic visits, QOL and NET symptom scores, laboratory assays including tumor marker(s), and triple-phase CT or MRI will be performed at 4-6 weeks posttreatment and then every 3 months \pm 4 weeks (Fig. 1).

\begin{tabular}{|c|c|c|c|c|c|c|c|}
\hline & Items & \begin{tabular}{|l|} 
Pre-enrollment \\
(- 1-2 wks)
\end{tabular} & $\begin{array}{l}\text { Intervention } \\
\text { period (1-4 } \\
\text { wks) }\end{array}$ & $\begin{array}{l}\text { Initial outcome } \\
\text { assessment (4-6 } \\
\text { wks) }\end{array}$ & $\begin{array}{l}\text { Follow-up } \\
\text { assessment } \\
\text { (every 8-16 wks } \\
\text { following initial } \\
\text { outcome } \\
\text { assessment) }\end{array}$ & $\begin{array}{l}\text { Final outcome } \\
\text { assessment ( } 24 \\
\text { months) }\end{array}$ & \\
\hline & $\begin{array}{l}\text { Inclusion } \\
\text { criteria }\end{array}$ & $\mathrm{x}$ & & & & & \\
\hline & $\begin{array}{l}\text { Exclusion } \\
\text { criteria }\end{array}$ & $\mathrm{x}$ & & & & & \\
\hline & $\begin{array}{l}\text { Informed } \\
\text { consent }\end{array}$ & $x$ & & & & & \\
\hline & $\begin{array}{l}\text { Clinical } \\
\text { evaluation }\end{array}$ & $\mathrm{x}$ & & $\mathrm{x}$ & $\mathrm{x}$ & $\mathrm{x}$ & \\
\hline & Lab assays & $\mathrm{x}$ & & $\mathrm{x}$ & $\mathrm{x}$ & $\mathrm{x}$ & \\
\hline & Embolotherapy & & $x$ & & & & \\
\hline & $\begin{array}{l}\text { QoL }^{1} \text { and } \mathrm{NET}^{2} \\
\text { symptom score } \\
\text { evaluation }\end{array}$ & & & $x$ & $x$ & $x$ & \\
\hline & $\begin{array}{l}\text { Triple phase CT } \\
\text { or MRI }\end{array}$ & $\mathrm{x}$ & & $x$ & $x$ & $x$ & \\
\hline & \begin{tabular}{|l|} 
Adverse event \\
evaluation
\end{tabular} & & & $x$ & $x$ & $x$ & \\
\hline & $\begin{array}{l}{ }^{1} \text { Quality of life (Qol } \\
{ }^{2} \text { Neuroendocrine } t\end{array}$ & $\begin{array}{l}\text { L) score based on E } \\
\text { umor (NET) symtpo }\end{array}$ & $\begin{array}{l}\text { RTC QLQ-C30 } \\
\text { s score base }\end{array}$ & $\begin{array}{l}\text { onnaire. } \\
\text {-NET21 module. }\end{array}$ & & & \\
\hline
\end{tabular}


If the patient remains within the eligibility criteria, repeat embolotherapy cycles can be performed when clinically indicated for intrahepatic progression. The second embolotherapy cycle will resume as described above. Total follow-up duration for each enrollee is at least 24 months. After this point, patients without disease progression will continue to receive clinical and imaging follow-up per institutional standard of care for the remaining duration of the trial.

\section{Study objectives \\ Primary endpoint}

The primary endpoint is HPFS from initial embolotherapy by Response Evaluation Criteria in Solid Tumors (RECIST) 1.0 criteria, as determined by blinded central review of follow-up imaging. Because all of the embolization techniques are in common clinical use, there will be no formal interim efficacy analysis.

\section{Secondary endpoints}

Secondary endpoints include comparisons of the following:

- Time interval between embolotherapy cycles among embolotherapy techniques

- NET symptom-free interval using the Carcinoid Symptom Severity Score among embolotherapy techniques

- Patient-reported quality of life (EORTC QLQ-C30) and NET symptom (G.I.NET21) scores among embolotherapy techniques

- Toxicity and adverse events among embolotherapy techniques

- Overall progression-free survival (PFS) by RECIST 1.1 criteria and duration of symptom control between gut and pancreatic NET, as well as among tumor grades

- Biomarkers (imaging, laboratory, and/or symptom) for treatment effect in all treatment arms

\section{Primary safety endpoint}

According to the Society of Interventional Radiology quality improvement guidelines for embolization and chemoembolization, major hepatic complications (biloma, abscess, liver failure) occur following approximately $6 \%$ of procedures, and the performance threshold is set at $8 \%$ [25]. The overall rate of any complication is $10 \%$, with a performance threshold of $15 \%$. All arms will be monitored for serious adverse events (SAEs) exceeding published thresholds. SAEs are classified as any event that is fatal; life-threatening; requires or prolongs hospital stay; results in persistent or significant disability, congenital anomaly, or birth defect; or an important medical event. Adverse events will also be classified using Common Terminology Criteria for Adverse Events
(CTCAE) grading. Liver function test elevations of CTCAE grade 3 or lower and any clinical symptoms attributable to postembolization syndrome will not be reported unless they meet a criterion. All toxicities/ events at or above CTCAE grade 4 will be reported. SAEs will be reported to the study sponsor by telephone and study form within 24 hours of the event. Interim safety analyses will be performed by an independent safety monitoring committee, which is composed of three interventional oncologists from institutions not involved in the trial, following accrual of 10 and 30 patients in each arm. A $20 \%$ rate of SAEs at these interim analyses will result in closure of the corresponding treatment arm.

\section{Statistical considerations \\ Sample size calculation}

Based on available retrospective data, median HPFS following BE is estimated at 11 months and CTACE at 20 months [9], corresponding to an HR of approximately 1.8. The effect of cTACE and DEB-TACE is presumed to be equivalent. Assuming a two-sided type I error rate of 10\%, 60 participants per arm will provide at least 92\% power for the overall log-rank test and $87 \%$ power for pairwise tests.

\section{Randomization}

The study will enroll 180 patients, who will be randomized in a 1:1:1 ratio to the three treatment arms. Randomization will be performed centrally and stratified by institution, using blocks of randomly selected sizes (3, 6 , or 9). Patients with symptomatic but not progressive liver disease will be monitored during the accrual phase to ensure balanced allocation between treatment arms, with adjustment as necessary.

\section{Trial time scale}

Preliminary experience from the pilot institution suggests that approximately six participants per site can be enrolled in 1 year. With at least 16 sites anticipated to participate in the study, a 24-month accrual period would result in approximately 180 enrollees. This provides an adequate margin for the target sample size of 194 patients, assuming a dropout rate of $8 \%$. Follow-up is a minimum of 24 months.

\section{Statistical analysis}

Our analysis will be aimed at identifying any embolotherapy arm that is significantly inferior (or superior) to the others based on comparison of the primary outcome. Our null hypothesis assumes that the arms are equivalent, without preconceived ranking. The test will use a studentized range approach developed for phase II screening trials using survival data [26, 27]. We will 
estimate the survival hazard rate in each treatment arm and compute the range of log-hazard rates (largest minus smallest). We will then compare the observed range to the distribution of the range under the null hypothesis. If the observed range exceeds the 95th percentile of its null distribution, any group whose hazard rate differs by more than this critical value from the group with the lowest PFS hazard will be declared significantly worse.

For secondary outcomes including symptom relief interval, time to reembolization, overall PFS, and PFS for subsets of the study sample, we will estimate the survival distribution using the Kaplan-Meier method. Comparisons among groups will be performed using log-rank tests, and survival regression models will be developed to estimate treatment effects, adjusted for baseline variables that may affect treatment outcomes.

For continuous outcomes (e.g., quality of life and symptom scores), summary statistics (mean, SD, 95\% CI) will be assessed for each of the study arms, and comparisons among arms will be performed using one-way analysis of variance and $t$ tests. Regression models will be used to estimate treatment effects adjusted for baseline variables that may affect treatment outcome. Binary variables (e.g., biomarkers) will be tabulated by treatment arm and compared using the chi-square test. Logistic regression models will be used to estimate adjusted effects.

\section{Data management and monitoring}

Data management will be handled by the Clinical Research Computing Unit at the University of Pennsylvania. Study data will be entered in an ORACLE database. Data entry will be performed by study coordinators and investigators at each of the sites.

Triphasic abdominal CT and MRI images will be uploaded to the Imaging Core at the American College of Radiology in Philadelphia and undergo central review by independent, blinded diagnostic radiologists unaffiliated with the participating institutions.

For patients who withdraw following embolotherapy, reasonable efforts will be made to track adverse effects, follow-up imaging results, and survival through the medical record at the treating institution by their treating physician.

Data quality checks have been built into the ORACLE data collection instruments. All database information will be encrypted, and de-identified data will be used for analysis. All investigators will permit study-related monitoring, audits, and inspections by the ethics committee, IRB, sponsor, government regulatory bodies, and university compliance and quality assurance groups of all study-related documents (e.g., source documents, regulatory documents, data collection instruments, study data). Remote audits of $5 \%$ of the data from each site will be perfumed at $50 \%$ accrual and at completion of accrual.

\section{Ethical considerations and dissemination}

The study protocol and any amendments will be submitted to a properly constituted independent ethics committee or IRB, in agreement with local legal prescriptions, for formal approval of the study conduct. The formal consent of a subject, using the ethics committee/IRB-approved consent form, must be obtained before that subject undergoes any study procedure. The consent form must be signed by the subject or legally acceptable surrogate and by the investigator-designated research professional. Unanticipated problems posing risks to subjects or others as noted above will be reported to the sponsor institution IRB.

Each subject is assigned a unique patient identification (PID) number in the ORACLE database. All research data and images are de-identified at the source and associated with the PID number.

The principal investigator is responsible for publication of the results of this study in whole or in part. Neither the complete nor any part of the results of the study carried out under this protocol, nor any of the information provided by the sponsor for the purposes of performing the study, will be published or passed on to any third party without the consent of the study sponsor. Any investigator involved with this study is obligated to provide the sponsor with complete test results and all data derived from the study.

\section{Discussion}

Despite several decades of experience with embolotherapy for NET liver metastases, there remains uncertainty regarding the optimal treatment modality and a paucity of level I evidence. Compared with other primary and metastatic liver malignancies, NET liver metastases have superior oncologic outcomes with a median survival of 18-65 months from initial embolotherapy [4-6, 9, 10, $12,20,21]$. The relatively indolent disease course and multimodality treatment approach have unique implications for determining the optimal liver-directed embolotherapy. The chosen technique should ideally not only achieve superior survival and local disease control but also have tolerable toxicity when repeated and used in combination with other therapies. The existing evidence base does not provide a clear answer regarding which embolotherapy best satisfies these criteria, reflected in lack of guideline recommendations specifying a specific embolotherapy technique [14-18].

The Randomized Embolization Trial for NeuroEndocrine Tumor Metastases to the Liver (RETNET) will be the first multicenter prospective RCT aimed at elucidating these questions regarding optimal embolotherapy 
technique. The results of this study will help refine the role of embolotherapy for NET liver metastases by determining which, if any, embolotherapy technique confers superior HPFS and/or safety profile between BE, lipiodol TACE, and DEB-TACE.

\section{Trial status}

Patient enrollment started in March 2017 and was ongoing at the time the manuscript was submitted. Completion of accrual is expected to take 24 months.

\section{Additional file}

Additional file 1: Standard Protocol Items: Recommendations for Interventional Trials (SPIRIT) 2013 checklist: recommended items to address in a clinical trial protocol and related documents, in addition to IRB approval letter. (DOCX $45 \mathrm{~kb}$ )

\section{Abbreviations}

BE: Bland embolization; CONSORT: Consolidated Standards of Reporting Trials; CT: Computed tomography; CTACE: Classical transarterial chemoembolization; CTCAE: Common Terminology Criteria for Adverse Events; DEB-TACE: Drug-eluting bead transarterial chemoembolization; EORTC: European Organization for Research and Treatment of Cancer; Gl: Gastrointestinal; HPFS: Hepatic progression-free survival; INR: International normalized ratio; IRB: Institutional review board; MRI: Magnetic resonance imaging; NET: Neuroendocrine tumor; PFS: Progression-free survival; PID: Patient identification; PRRT: Peptide receptor radionuclide therapy; QOL : Quality of life; RCT: Randomized controlled trial; RECIST: Response Evaluation Criteria in Solid Tumors; RETNET: Randomized Embolization Trial for NeuroEndocrine Tumor Metastases to the Liver; SAE: Serious adverse events; SPIRIT: Standard Protocol Items: Recommendations for Interventional Trials; TACE: Transarterial chemoembolization; WHO: World Health Organization

\section{Funding}

This study is funded by a grant to the University of Pennsylvania from Guerbet LLC (Princeton, NJ, USA).

\section{Authors' contributions}

JXC is the primary author of this manuscript, which describes a clinical trial designed and written by MCS and PW. All authors read and approved the final manuscript.

\section{Ethics approval and consent to participate}

This study was approved by the University of Pennsylvania Institutional Review Board (IRB) and the IRB or corresponding ethics committee at each participating site.

\section{Consent for publication}

Not applicable.

\section{Competing interests}

MCS has received research funding from Guerbet LLC and BTG international and is a consultant for Guerbet LLC, Merit Medical, Terumo Medical, and Bayer/Onyx

The remaining authors declare that they have no competing interests.

\section{Publisher's Note}

Springer Nature remains neutral with regard to jurisdictional claims in published maps and institutional affiliations.

\section{Author details}

${ }^{1}$ Division of Interventional Radiology, University of Pennsylvania, Philadelphia, PA, USA. ${ }^{2}$ Department of Epidemiology \& Biostatistics, University of Pennsylvania, Philadelphia, PA, USA. ${ }^{3}$ Abramson Cancer Center, University of Pennsylvania, Philadelphia, PA, USA. ${ }^{4}$ Department of Radiology, Hospital of the University of Pennsylvania, 1 Silverstein, 3400 Spruce Street, Philadelphia, PA 19104, USA.

Received: 8 December 2017 Accepted: 4 July 2018

Published online: 17 July 2018

References

1. Yao JC, Hassan M, Phan A, Dagohoy C, Leary C, Mares JE, Abdalla EK, Fleming JB, Vauthey JN, Rashid A, Evans DB. One hundred years after "carcinoid": epidemiology of and prognostic factors for neuroendocrine tumors in 35,825 cases in the United States. J Clin Oncol. 2008;26:3063-72.

2. Chamberlain RS, Canes D, Brown KT, Saltz L, Jarnagin W, Fong Y, Blumgart LH. Hepatic neuroendocrine metastases: does intervention alter outcomes? J Am Coll Surg. 2000;190:432-45.

3. Barbier CE, Garske-Roman U, Sandstrom M, Nyman R, Granberg D. Selective internal radiation therapy in patients with progressive neuroendocrine liver metastases. Eur J Nucl Med Mol Imaging. 2016;43:1425-31.

4. Dong XD, Carr Bl. Hepatic artery chemoembolization for the treatment of liver metastases from neuroendocrine tumors: a long-term follow-up in 123 patients. Med Oncol. 2011;28(Suppl 1):S286-90.

5. Fiore F, Del Prete M, Franco R, Marotta V, Ramundo V, Marciello F, Di Sarno A, Carratu AC, de Luca di Roseto C, Colao A, Faggiano A. Transarterial embolization (TAE) is equally effective and slightly safer than transarterial chemoembolization (TACE) to manage liver metastases in neuroendocrine tumors. Endocrine. 2014;47:177-82.

6. Gupta S, Johnson MM, Murthy R, Ahrar K, Wallace MJ, Madoff DC, MCRae SE, Hicks ME, Rao S, Vauthey JN, et al. Hepatic arterial embolization and chemoembolization for the treatment of patients with metastatic neuroendocrine tumors: variables affecting response rates and survival. Cancer. 2005;104:1590-602

7. Memon K, Lewandowski RJ, Riaz A, Salem R. Chemoembolization and radioembolization for metastatic disease to the liver: available data and future studies. Curr Treat Options in Oncol. 2012;13:403-15.

8. Pericleous M, Caplin ME, Tsochatzis E, Yu D, Morgan-Rowe L, Toumpanakis C. Hepatic artery embolization in advanced neuroendocrine tumors: efficacy and long-term outcomes. Asia Pac J Clin Oncol. 2016;12:61-9.

9. Ruutiainen AT, Soulen MC, Tuite CM, Clark TW, Mondschein Jl, Stavropoulos SW, Trerotola SO. Chemoembolization and bland embolization of neuroendocrine tumor metastases to the liver. J Vasc Interv Radiol. 2007;18:847-55.

10. Sofocleous CT, Petre EN, Gonen M, Reidy-Lagunes D, Ip IK, Alago W, Covey AM, Erinjeri JP, Brody LA, Maybody M, et al. Factors affecting periprocedural morbidity and mortality and long-term patient survival after arterial embolization of hepatic neuroendocrine metastases. J Vasc Interv Radiol. 2014;25:22-30. quiz 31

11. Sommer WH, Ceelen F, Garcia-Albeniz X, Paprottka PM, Auernhammer CJ, Armbruster M, Nikolaou K, Haug AR, Reiser MF, Theisen D. Defining predictors for long progression-free survival after radioembolisation of hepatic metastases of neuroendocrine origin. Eur Radiol. 2013;23:3094-103.

12. Strosberg JR, Choi J, Cantor AB, Kvols LK. Selective hepatic artery embolization for treatment of patients with metastatic carcinoid and pancreatic endocrine tumors. Cancer Control. 2006;13:72-8.

13. Bhagat N, Reyes DK, Lin M, Kamel I, Pawlik TM, Frangakis C, Geschwind JF Phase II study of chemoembolization with drug-eluting beads in patients with hepatic neuroendocrine metastases: high incidence of biliary injury. Cardiovasc Intervent Radiol. 2013;36:449-59.

14. Boudreaux JP, Klimstra DS, Hassan MM, Woltering EA, Jensen RT, Goldsmith SJ, Nutting C, Bushnell DL, Caplin ME, Yao JC, North American Neuroendocrine Tumor Society (NANETS). The NANETS consensus guideline for the diagnosis and management of neuroendocrine tumors: welldifferentiated neuroendocrine tumors of the jejunum, ileum, appendix, and cecum. Pancreas. 2010;39:753-66.

15. Kennedy A, Bester L, Salem R, Sharma RA, Parks RW, Ruszniewski P, NETLiver-Metastases Consensus Conference. Role of hepatic intra-arterial therapies in metastatic neuroendocrine tumours (NET): guidelines from the NET-Liver-Metastases Consensus Conference. HPB (Oxford). 2015;17:29-37.

16. Kulke MH, Anthony LB, Bushnell DL, de Herder WW, Goldsmith SJ, Klimstra DS, Marx SJ, Pasieka JL, Pommier RF, Yao JC, et al. NANETS treatment guidelines: well-differentiated neuroendocrine tumors of the stomach and pancreas. Pancreas. 2010;39:735-52.

17. Kulke MH, Shah MH, Benson AB 3rd, Bergsland E, Berlin JD, Blaszkowsky LS, Emerson L, Engstrom PF, Fanta P, Giordano T, et al. Neuroendocrine tumors, version 1.2015. J Natl Compr Cancer Netw. 2015;13:78-108. 
18. Pavel M, Baudin E, Couvelard A, Krenning E, Oberg K, Steinmuller T, Anlauf M, Wiedenmann B, Salazar R, Barcelona Consensus Conference participants. ENETS Consensus Guidelines for the management of patients with liver and other distant metastases from neuroendocrine neoplasms of foregut, midgut, hindgut, and unknown primary. Neuroendocrinology. 2012;95:157-76.

19. Pitt SC, Knuth J, Keily JM, MCDermott JC, Weber SM, Chen H, Rilling WS, Quebbeman EJ, Agarwal DM, Pitt HA. Hepatic neuroendocrine metastases: chemo- or bland embolization? J Gastrointest Surg. 2008;12:1951-60.

20. Maire F, Lombard-Bohas C, OToole D, Vullierme MP, Rebours V, Couvelard A, Pelletier AL, Zappa M, Pilleul F, Hentic O, et al. Hepatic arterial embolization versus chemoembolization in the treatment of liver metastases from well-differentiated midgut endocrine tumors: a prospective randomized study. Neuroendocrinology. 2012;96:294-300.

21. Ho AS, Picus J, Darcy MD, Tan B, Gould JE, Pilgram TK, Brown DB. Long-term outcome after chemoembolization and embolization of hepatic metastatic lesions from neuroendocrine tumors. AJR Am J Roentgenol. 2007;188:1201-7.

22. Guiu B, Deschamps F, Aho S, Munck F, Dromain C, Boige V, Malka D, Leboulleux S, Ducreux M, Schlumberger M, et al. Liver/biliary injuries following chemoembolisation of endocrine tumours and hepatocellular carcinoma: lipiodol vs. drug-eluting beads. J Hepatol. 2012;56:609-17.

23. Gaba RC. Chemoembolization practice patterns and technical methods among interventional radiologists: results of an online survey. AJR Am J Roentgenol. 2012;198:692-9.

24. Vinik E, Silva MP, Vinik Al. Measuring the relationship of quality of life and health status, including tumor burden, symptoms, and biochemical measures in patients with neuroendocrine tumors. Endocrinol Metab Clin N Am. 2011;40:97-109. viii

25. Gaba RC, Lokken RP, Hickey RM, Lipnik AJ, Lewandowski RJ, Salem R, Brown DB, Walker TG, Silberzweig JE, Baerlocher MO, et al. Quality improvement guidelines for transarterial chemoembolization and embolization of hepatic malignancy. J Vasc Interv Radiol. 2017;28:1210-1223.e3.

26. Liu PY, Moon J, LeBlanc M. Phase II selection designs. In Crowley J, Ankerst DP, editors. Handbook of statistics in clinical oncology. 2nd ed. Boca Raton, FL: Chapman \& Hall/CRC; 2006. p. 155-66.

27. Liu P, Dahlberg S, Crowley J. Selection designs for pilot studies based on survival. Biometrics. 1993;49:391-8.

Ready to submit your research? Choose BMC and benefit from:

- fast, convenient online submission

- thorough peer review by experienced researchers in your field

- rapid publication on acceptance

- support for research data, including large and complex data types

- gold Open Access which fosters wider collaboration and increased citations

- maximum visibility for your research: over $100 \mathrm{M}$ website views per year

At $\mathrm{BMC}$, research is always in progress.

Learn more biomedcentral.com/submissions 